The Lasting Effects of Growing up in a Military-Connected

Home: A Qualitative Study of College-Aged

American Military Kids

Taylor E. Zurlinden ${ }^{1 *}$ [hobbsta17@students.ecu.edu]

Michael W. Firmin²[firmin@cedarville.edu]

Aubrey L. Shell ${ }^{3}$ [aubgille@iu.edu]

Hannah W. Grammer ${ }^{2}$ [hannahfoster@cedarville.edu]

${ }^{1}$ East Carolina University, Department of Psychology, Greenville, NC, USA

${ }^{2}$ Cedarville University, Department of Psychology, Cedarville, OH, USA

${ }^{3}$ Indiana University-Purdue University Indianapolis, Department of Psychology, Indianapolis, IN, USA

Correspondence can be directed to:

Taylor E. Zurlinden

Department of Psychology, Rawl Building, East Carolina University, Greenville, NC 27858.

Phone: 252.328 .6800

E-mail: hobbsta17@students.ecu.edu

This is the author's manuscript of the article published in final edited form as:

Zurlinden, T. E., Firmin, M. W., Shell, A. L., \& Grammer, H. W. (2019). The lasting effects of growing up in a militaryconnected home: A qualitative study of college-aged American military kids. Journal of Family Studies, 0(0), 1-17. https://doi.org/10.1080/13229400.2019.1650800 


\begin{abstract}
Being reared as a military kid is a unique experience shared by millions of children all over the world. This study seeks to understand the experiences shared by military kids and how those experiences change their perspectives, behaviors, and relationships. In the present phenomenological study, we conducted 21 in-depth, semi-structured qualitative interviews of college students who were reared as United States military kids (MKs). Using criterion sampling, the participants all met specific criteria, including the deployment of their military parent during their lifetime, as well as a minimum of 10 years of parental service during the MK's lifetime. Participants represented four of the five major military branches. Interviews were tape recorded, transcribed, and coded for analysis. Researchers reviewed the transcribed interviews to find common themes and meta-themes present in a majority of the interviews. Common themes generally centered around relationships with family, changes during childhood, and military presence in everyday life. Particular findings reported in the present article are regarding demonstrated respect, military pride, military community commonality, anticipation of change, childhood instability, family dynamics, military terminology, adaptability, and an overall positive experience. These common themes are relevant for all involved in the care of military kids and their families as they underline the unique perspective that MKs have about their childhood and their future.

Key words: Parental Deployment; Military Kids; Family Relationships; Family Dynamics; Development
\end{abstract}




\section{The Lasting Effects of Growing up in a Military-Connected Home: A Qualitative Study of College-Aged American Military Kids}

Children who are reared in households with a parent serving in the military often face challenges that other children do not, such as parental deployment and frequent relocations. These individuals gain unique experiences that affect their perceptions on life, including the possibilities of living in military housing, increased traveling due to the parent's deployment locations, different parenting techniques, and worry about the deployed parent's safety (Flake, Davis, Johnson, \& Middleton, 2009). Mustillo, Wadsworth, and Lester (2016) reported these relationship dynamics to be particularly impactful with military kids (MKs). In 2013, almost 1.2 million US children were a part of families with service members. Of that number, approximately 250,000 children were in each respective age group: 0 to 2,3 to 5 , and 6 to 10 years of age (Office of the Deputy Assistant Secretary of Defense, 2013). Because these ages are each highly important in children's development, disruptions of a parent leaving the family for deployment can increase negative behavioral symptoms (Chartrand, Frank, White, \& Shope, 2008). Furthermore, deployment sometimes has been connected with increased anxiety compared to community norms, as well as both externalizing (such as aggression and rulebreaking) and depressive symptoms in children ages six to 12 (Lester et al. 2010). Furthermore, the 2006 Survey of Active-Duty Spouses (Defense Manpower Data Center, 2006) reported that $20 \%$ of MK parents believed that their child coped poorly during parental deployment, which provides evidence that the issues are noticeable in the home as well.

Previous research has also focused on the timing and length of deployements, and how they may affect development. Mustillo et al. (2016) conducted 680 telephone interviews, followed by a web-based survey. They reported that children were exposed to deployment 
between $16 \%$ and $20 \%$ of their lives across all of the age groups studied. Children between the ages of three to five who experienced a recent parental long-deployment experienced elevated levels of generalized anxiety. Researchers also found that, with each 1-month increment in age of child, the risk for problematic development increased by $3 \%$. The total percentage of life exposed to deployment was associated with greater reported social anxiety in the same age group. For older children who ranged from 6-10 years in age, having a parent deployed at birth was associated with a reported detriment to emotional and relational problems with peers. In a separate study, Gorman and colleagues (2010) found an 18\% increase in stress disorders and $19 \%$ increase in behavioral issues in children who currently had a parent deployed. Overall, they found an $11 \%$ increased likelihood of hospital visits for both mental and physical issues. These research findings showed overall higher reports of stress among military children. Additionally, these studies emphasize that parental deployment can have an immediate impact on a child's mental and physical health, however, the long-term effects are not known. Although these studies are not representative of all MKs, the findings do suggest a potential link between the time a parent is deployed and the psychological effects it may have on the child.

Unfortunately, even when deployed parents return home, reintegration into the family can be challenging. Missing significant time spans of a child's life and development can have negative impacts on the relationship (Clever \& Segal, 2013). Sandoz, Moyer, and Armelie (2015) explored post-deployment interactions, potential family reintegration problems, and the implications of overcoming (or not overcoming) such problems. While some cases of postdeployment reintegration were shown to be challenging for family units, researchers also found that, when veterans were willing to actively engage in individual therapy for mental health concerns related to the deployment, they were able to experience personal and relational growth. 
Alfanoa and colleagues (2016) similarly reported that a uniform pattern does not exist for all MKs, nor do all such individuals frame their own experiences similarly. Rather, the children's idiosyncratic and specific circumstances, including parental mental health, support, and contextual factors appear to impact overall outcomes regarding most MKs.

While there have been several large-scale studies of military kids, both qualitative and quantitative, there is a general lack of research on military kids once they are outside of the home and enrolled in college. Although the research shows the unique struggles that military kids may face during a parent's deployment, and immediately following homecoming, there is little research about the long-term impact of these deployment's on the child's development, especially from the MKs' perceptive. Additionally, few studies report on the potential positive benefits from being reared in a military family.

Particularly because context plays such an important factor regarding the outcomes of MKs' experiences, we utilized semi-structured interview, qualitative methodology in our present study. While quantitative research typically answers "what" or "how many" types of research questions, qualitative methods more often answer "why" or "how" types of research questions (Creswell, 2012). We were most interested in understanding potential dynamics that MKs experience and how they come to frame their understanding of their developmental years as MKs. This research seeks to expand on the existing literature and explore both the negative and positive consequences of having a parent in the military. The MKs' personal perception about how their parent's deployment impacted their development and has led to their current state is of particular interest. As such, these overarching research questions drove the selection of our research protocol, and a desire to listen to and learn from an underrepresented sample: collegeaged MKs. 


\section{Method}

\section{Procedure}

Because we inductively sought to understand the life of MKs from their own perspectives, the present research design was a phenomenological study, which aimed to understand the shared, lived experience of the target population (Sin, 2010). The interviews were conducted by three members of the present research team and without additional research assistants. The interviews were conducted in a dedicated portion of the psychology department's qualitative research lab. Before beginning the interviews, participants were given information about our study, such as the study's purpose, potential risks and benefits, and implications. Participants were also informed that they would not be compensated for their time, and that participation was voluntary. Due to the sensitive nature of the material discussed, confidentiality was also emphasized with all participants. Participants were then asked to sign an informed consent document expressing their full understanding of our study.

We conducted semi-structured qualitative interviews (Alvesson, 2011). We chose this protocol so that participants possessed freedom to answer interview questions as fully as they desired, telling personal stories and elaborating on topics as they saw fit. While specific interview questions varied among participants (i.e., they were semi-structured), all of the interviews focused on the perceived effects that being reared as MKs, particularly with regards to parental deployment, had on the participants' affect, behavior, and cognition. Allowing variation between subjects provided researchers the freedom to probe particular topics further with individual participants, while also allowing for discretion when a participant appeared uncomfortable by a particular question. 
Interviews were digitally recorded and later transcribed by the researchers. After transcription, we utilized an open-coding data analysis method (Maxwell, 2012), applying an inductive approach for themes to arise from the interviews. We applied constant comparison analysis (Silverman, 2012) of the respective transcripts, examining common words, phrases, and concepts among the interviews. Each researcher possessed copies of all the interview transcripts and engaged in independent data-driven coding, using a line-by-line assessment of the data (Chenail, 2012a). During this period, a codebook was created to help organize the commonly occurring words, phrases, and ideas that appeared in the interviews (DeCuir-Gunby, Marshall, \& McCulloch, 2011). Creating a codebook allows researchers to create inclusion and exclusion criteria for each of the codes, and organize key examples of the codes. After individual analysis, we collaborated to assess which codes were most common and represented a consensus among the research team.

Following the example of Gay, Mills, and Airasian (2009), we asked key questions, conducted organizational review, visually displayed the potential findings, and employed concept mapping as qualitative research techniques in order to move from coding to generating the themes reported in our present study. All reported themes represent the consensus among all our study's authors.

Internal validity, or credibility, of the research study was strengthened through a number of protocols. One was that the research team met regularly in order to discuss the themes being generated among the respective researchers. While such collaboration does not assure credibility, the process does generally support confidence that the themes being reported in a study aptly reflect the consensus of the research participants, as researchers must have evidence from multiple interviews to support their proposed themes (Rapport et al. 2013). These frequent 
meetings also helped researchers consider possible biases when interpreting data and ensured that the interpretation of the data was not skewed by the researchers' personal outlooks. Recognizing and controlling for personal biases is one of the key features of phenomenological research (Moustakas, 1994).

Second, after having generated the themes reported in the present article, we subsequently comprised a data audit (Rodgers, 2008). This involved combing through the entirety of the recorded transcripts and locating participant quotations that supported each of the reported themes. Utilizing a data trail helped ensure that sufficient data supports the themes identified in a study, enables independent researchers to examine our study and decipher potential fraud (if it should exist), and aids future researchers who wish to extend the study with additional samples. Third, we utilized the expertise of an independent researcher (Flick, 2006). This was an individual possessing a national reputation in qualitative research who reviewed each aspect of the research process, and checked the quality of each phase involved in our study. The independent researcher also inspected the data audit, believing that sufficient data exists in order to justify the conclusions drawn and reported in the present article.

Fourth, member checking (Mero-Jaffe, 2011) helped to strengthen our study's trustworthiness and credibility. To member-check, we went back to MKs in the sample and shared with them the findings being reported in the present study. All were in substantial agreement that the conclusions we drew are fitting representations of their respective sentiments. Fifth, when we generated the present manuscript for publication, we utilized low inference descriptors (Chenail, 2012b). This qualitative protocol helps strengthen a study's trustworthiness and credibility because it forces authors to ground their reported conclusions in actual words of the research participants, helping to ensure that sentiments are being represented rightly. Finally, 
we achieved saturation (O'Reilly \& Parker, 2012) that strengthened our confidence that the sample size was adequate to accomplish our research aims. In particular, we consistently found that the same themes reoccurred among the various transcripts (i.e., adding new interviews did not add new potential themes). Consequently, consistent with qualitative experts such as Guest, Bunce, and Johnson (2006), Neuman (2006), and Oppong (2013), we believe the sample size was sufficient in order to aptly draw the conclusions that we report in the present article.

In sum, internal validity, or credibility, is a grounding principle for a rigorous qualitative research study (Newman, Ridenour, Newman, Smith, \& Brown, 2013). It bolsters confidence that the findings we report in our present study are adequate representations of the perceived worlds of the participants being studied. We believe that our study's design and implementation represents the rigor expected of solid scholarship by established qualitative research standards (Sin, 2010). This study was approved by the University's IRB, and adhered to strict ethical and scientific guidelines.

\section{Participants}

The sample of MKs in our present study consisted of volunteers from a private, comprehensive, Midwestern University. We sent a campus-wide e-mail message, asking students to self-identify if they had been reared as a MK; over 150 replies were returned. From this initial group, we used criterion sampling (Berg, 2012), otherwise known as purposeful sampling (Harsh, 2011), in order to select a pool of potential participants. A follow-up message was sent to the 150 students in order to winnow the group in accordance with our criteria of research interest. Subsequently, 20 Caucasian students, and one Hispanic student, agreed to participate in our study, with all participants meeting the following criteria: (a) at least one parent who served in the military, (b) the parent(s) had been in active duty for at least 10 years of the child's life, 
and (c) the parent(s) must have been deployed for at least two months during the child's developmental years. These criteria were established in order to create a homogenous sample, increasing the likelihood of generating themes in the data set (Firmin, 2006). As such, we desired to utilize a sample in which all participants underwent similar experiences in relation to military upbringing. In particular, we chose the ten-year criterion in order to ensure that a significant portion (approximately half) of the MKs life was spent as an active duty MK.

A team of three researchers interviewed all 21 students; 13 were female and 8 were male, with ages ranging from 19 to 22 . Of the participants, all 21 had a father who was in the military, and one had both parents serve in the military. Of note however, the participant with both parents in the military described a paternal deployment, and did not mention a maternal deployment. Participants had moved an average of six times during their parents' military career. The longest time the MKs lived in one place averaged approximately five years; several participants stated that their time at college (four years or fewer) was the longest that they had ever lived in one place. Twelve of the participants lived in another country during their parent's military service. The military branches represented by the participants were Army [6], Navy [1], Air Force [12], Marine Corps [1], and one participant did not disclose the specific military branch. The high concentration of Air Force kids is likely due to the campus' proximity to a large Air Force base located nearby. At the time of data collection, three of the participants currently served in the military through the Reserve Officers' Training Corps programs (Army and Air Force) located on campus. Names used in the present article are pseudonyms and are intended to enhance readability.

\section{Findings}


The findings presented in the present article are common themes that were reported by most of the research participants. As such, we believe the reported findings are generally representative of the sample as a whole, and accurately describe the perspectives of the interviewed MKs. Common themes generally centered around relationships with family, changes during childhood, and military presence in everyday life. In the present article, we use the term “civilian" to refer to anyone who was not directly connected to military service.

\section{Demonstrated Respect}

Participants in our study consistently stressed the importance of respect that was expected to have been demonstrated in their households during their developmental years. They also stated that their military parent emphasized obedience to, and respect for, authority. The word "respect" was used throughout the interviews in regards to several different aspects of the participants' upbringing. When asked about their views regarding respect compared to their civilian peers several participants widened their eyes, smirked, and let out a little laugh. They continued to explain that giving respect was never an option in their home and that they learned about veneration very early on. Some participants described a "hierarchy" or "chain" of respect within the household and, as children, they were expected to be respectful to both adults and peers. In reference to learning about respect at an early age, for example, Christina summarized:

We were very respectful kids, just because we grew up in that environment, my parents taught us, like my dad called everybody "sir" so we call everybody "sir" and "ma'am." So we just had that instilled in us from the very beginning.

Most participants echoed Christina's idea of the military parent serving as a model for respect. Due to the very hierarchical structure of military authority, participants indicated that 
they were given ample opportunities to see their parent both give and receive respect from others. Participants also shared that their parents provided a role-model of how to show respect to those in authority, despite personal opinions. Regardless of whether or not the participants agreed with what the authorities were saying, they nonetheless were expected to speak and behave in a manner that explicitly communicated respect. This mindset was said to have been carried over into the participants' adult lives; participants shared that they believed themselves to be much more respectful of the university's rules than their civilian-reared peers. Several participants shared specific instances during their education where they chose to be respectful, despite personal disagreements with university policy and/or various college personnel. The participants stressed the importance of rules and emphasized the value which rules hold in a society. Overall, participants felt that they had been trained more fully regarding the importance of respect and, as a result, they were more respectful toward authority than were their civilian counterparts.

\section{Military Pride}

Most participants used the word "pride" in their descriptions regarding how they feel about their upbringing as MKs. Participants described feeling dignity in their identity as a MK and the connotations that the title held. Participants further described feeling that their identity as a MK resulted in having had experiences that most of their civilian peers were not provided. Haylie excitedly stated the sentiments of most participants in this regard: "I think it's just really cool being able to say that I've traveled, I've been to different places in the world, and yeah I'm just really proud of being a military kid."

Another major domain in which participants consistently described feeling proud of the service that their military parent provided to the USA. MKs described themselves as feeling 
honored that their parent has sacrificed for his/her country and fought for America's freedom. They described feeling specific honor for the determination and bravery of their parent, and in general for what military service represents. As one of many participant examples, Trevor became teary as he described, “... all the thank yous, that just puts pride in my dad and pride in being my dad's kid and in the military that's making a difference or protecting our country." Overall participants felt honored regarding not only their identity as MKs, but also their parent's military service. The dignity was described in terms of both who they were and what their parent(s) did.

\section{Military Community Commonality}

Another common theme among participants was a strong sense of belonging in the military community. Words such as "bond," "commonality," "support," “connection," and "understanding" were frequently used when referring to the military community. Participants said that they often felt instantly connected to an individual when they learned of the other person's military connection. Often times, the MKs formed a quick bond and began sharing stories (including frustrations) together. In particular, participants said they felt that other MKs best understood their lifestyle and personality elements (e.g., respect for authority). The MKs also disclosed that many times they felt that their most enduring friendships were with other MKs. Daniel described the sentiments of most participants when he shared: "I think you just kind of lean towards $[\mathrm{MKs}]$ because they know you and you know them... they know what it's like, you know what it's like."

Although participants expressed feeling as though they were constantly "new kids" and seldom felt like "locals," they routinely felt accepted among their military peers. The MKs stated that, although their civilian peers often felt hurt by the frequent moves (i.e., due to lost 
friendships), other MKs better knew how to handle leaving friends and being left behind. This sense of understanding was clearly articulated by Sarah, who admitted: "[The base school had] a better sense of belonging because [we] were with [our] people." As such, although participants possessed diverse backgrounds, such as different branches, ranks, locations, and experiences, they seemingly found a commonality within the military community. Callie summarized the overall sentiment of participants finding community in the military: "Even though I don't know everyone, military is family, they take care of everyone...." We note that several participants stated a desire to connect with more MKs on the college campus and also an eagerness to hear the findings regarding our study study (i.e., presumed validation that they were not alone).

\section{Childhood Instability}

A fourth theme that emerged from the interviews with MKs was an acute awareness that life could potentially change significantly at any point in time. Participants shared that frequent life changes caused them to feel less comfortable in their respective environments and that this dynamic had a clear impact on their social lives. One participant illustrated her sentiments regarding this point by noting that, when growing up, her homes routinely had relatively few decorations; her family reportedly never had a chance to really "settle" into a home before their military parent was transferred. Another participant shared that he often was not fully comfortable in his housing situations due to frequent transfers. Some participants shared that the moves became so common, they now feel somewhat restless while at college, because it is a stable, four-year living experience. One student who was nearing the end of her education stated that she was excited to leave school so that she could travel and explore once more.

A repeated sentiment regarding this theme, which was echoed by a majority of participants, concerned the perceived effects that constant moves had on friendships. Participants 
made frequent references to both "quick" and "shallow" friendships. Although they reportedly made friends everywhere they went, the MKs also felt that the friendships were qualitatively different than friendships most often experienced by civilians. Anna summarized the sentiments of most MKs in our study when she regretfully stated:

Change is a constant... so that's changed me emotionally: I don't put down roots as deep. I put quick shallow roots often times, and not just really deep slow growing roots.... Because, if you have too deep of roots, when you pull them up-it just hurts.

Due to the frequent moves and sudden changes in lifestyle, participants repeatedly mentioned experiencing a lack of "lifelong" constants, including having no place that they considered to be a hometown. Participants moved an average of six times during their parent's military career, so they were uprooted frequently during their developmental years. The median longest length of stay in a single location was four years. This construct was especially noted by several participants who indicated that college was their longest continual residence. In general, participants shared that they had a difficult time answering the question, "Where are you from?," because they did not have a typical hometown. This dynamic became more obvious to participants when they assessed their lives in the long-term, and realized that their roots were not deep in any one location. For example, Kylie admitted:

I think I don't have the attachment to one place that I would expect probably most people would, if they were growing up in the same place. I think I've gotten it more since I've been here because I've lived here the longest. 
Several participants shared that, when growing up, they were unaware that some people had "best friends since birth." They could not imagine what it would be like to have had a consistent peer group during childhood, as Sarah noted: “Oh my gosh, it's not just in movies; like people actually have friends that they've had their entire life." Many participants shared that, although they did make friends at their different locations, the MKs rarely maintained those relationships long-term. While they had numerous new friends in various locales, the participants indicated that they tended not to maintain those friendships once they moved to a different location.

\section{Family as a Constant}

Due to the perpetual relocations, participants shared that they felt a need for something that would remain stable and steady amidst the variability. MKs in our study related that family was the constant in their lives, regardless of their locations or circumstances. One participant stated this point directly by saying: "Moving a lot, we were looking for something that was stable, and family is." Even though life for the MKs was often unpredictable, participants reportedly felt as though they could always rely on their families. Rachel stated the sentiments of most participants when indicating: "At the end of the day, it's your family that's the ones that's been with you." As such, the family unit evidently was perceived as the bedrock and constant on which the participants drew feelings of security. In particular relationships with their siblings and mothers were described as being changed in a positive, long-term way by their military parent's deployment.

Siblings. Participants frequently stated that they were especially close with their sibling(s). In general, participants felt that, regardless of where they moved, they always knew that they would have friends in their sibling(s); the MKs also said they felt it was important to 
maintain good relationships with their sibling(s) for this reason. One participant even seemed disgusted by the fact that some people did not maintain deep relationships with their brothers/sisters. When asked whether or not they currently felt close with their sibling(s), participants universally responded with an overwhelming "YES!"

At a deeper level, the MKs in our study related having felt a reliance on sibling(s) during parental deployment. Brothers/sisters seemed to realize the importance of generally being cooperative, rather than antagonistic, during deployments periods. With one less parent in the home, MKs said that they came to rely on their sibling(s) more during the deployment. They knew that their brothers/sisters understood their circumstances — as if they were fighting along with them on the same side of a battle. During deployments, their civilian peers reportedly were often unable to understand the MKs emotional pain, so siblings were left to rely on each other for social support. Similar to the other participants, Kylie summarized the effect of her father's deployment on her and her sibling(s)' relationship: "I think we definitely stuck closer together; we valued each other more."

Participants also frequently described bearing more responsibility to serve as a leader and role model for their siblings during deployments. Female participants commented that during deployments they sometimes felt as though they became a second mother to their younger sibling(s). Male participants similarly stated that they recalled having felt as though they assumed the proverbial role of "Man of the House" during their father's absence. Jonathan shared his thoughts, and also echoed the thoughts of the other male MKs, when he stated:

They would definitely look up to me. That made me to remember to do the right thing, because they're watching [me], they don't have a father figure, and my 
dad always told me when he left, "You're them man of the house now." So I knew that I had to show my brother some type of way to live and way to love. According to our participants, these changes in roles and relationships created permanent changes in the sibling dynamics.

Mothers. A common sentiment among the participants was that MKs felt closely connected with their mothers because of the military lifestyle (e.g., work trips, deployments, and training) that often took their father away from the family. One participant described her childhood by saying that she felt essentially only raised by her mom. Several participants went as far as to say that they could empathize with children raised by single-moms because of their unique upbringing.

Another aspect of mother-child relationships that appeared frequently among the MKs was a better understanding of their mother as a real and vulnerable person. Due to the increased responsibility that older children undertook during deployments, they began to better understand how much of a burden their mothers were carrying. Participants described various situations when they saw how hurt and/or scared their mother was. One MK even shared that her mom refused to sleep alone in her bed while her father was gone and, instead, spent nine months sleeping on the couch. Participants frequently described that, because of how stressed they saw that their mother was, they stepped-up more than usual in order to bear more responsibility. This dynamic reportedly led to participants often referring to their relationships with their mother as a "partnership" and "team effort."

Participants in our present study also identified a theme of experiencing difficulty reintegrating the deployed parent back into the home following deployment. The most noticeable difficulties came as the family tried to return to their "pre-deployment roles," after 
the parent returned. Due to their father being gone, MKs were left to rely on their mother as the sole parent during deployments. When the father returned home from having served overseas, some participants found the reintegration somewhat challenging, because they had learned not to rely on him while he was out of the country and had, instead, come to depend on their mother. Participants acknowledged that they had experienced relatively unique relationships with their mothers due to the deployment and expressed this dynamic in a variety of ways, including the use of phrases such as, "I gravitate towards my mother," and "I am more drawn to my mother." Although deployments were for limited time-frames, the accompanying dynamics created lasting impacts on how MKs related to their mothers. Generally the participants in our sample expressed feeling as though the military lifestyle helped MKs appreciate the relationship with their mothers more, and also to value her in qualitatively different ways than their civilian peers valued their mothers.

\section{Military Terminology}

MKs frequently used acronyms and military terms when engaging in the semi-structured interviews. Terms such as MOS (military occupational specialty), XO (executive officer), TDY (temporary duty) and CO (commanding officer) were all used amidst their regular language. We also noticed that participants frequently used military-themed terms. For example, MKs in our study often referred to the family as a "unit," making statements such as, "we function as a unit," and "we move as a unit." Participants were aware that many of the terms and phrases they used were not common among the civilian population, as they occasionally took the time to explain the terms to us as researchers. Military vocabulary was described in terms as having been a bonding language of MKs: they literally understood one another on a deeper level. Similarly, participants frequently referenced humor or phrases that might be considered rude if stated by a 
civilian. Examples of these include using terms such as "military brat" and "Chair Force." We noted that participants varied, not only in their definitions of the term "military brat," but also regarding their attitude towards that term. Participants with parents in all branches made reference to the "Chair Force" moniker given to the Air Force, while no participant used the term maliciously.

\section{Adaptability}

The MKs in our study indicated feeling that they possessed more adaptability than did their civilian peers. Participants frequently used words such as "flexible," "adaptable," "understanding," and "willing." Due to the frequent moves and necessary accompanying life adjustments, MKs felt that they had become more flexible to changes that life required of them. In general, participants in our study talked about how their past experiences have affected their current behaviors. MKs frequently referred to daily challenges and how their mindsets often differed from their civilian peers' mindsets, due to their unique developmental experiences. Current, daily challenges were said to be perceived as being less overwhelming, when compared to major life adjustments with which MKs had to deal during childhood. This past adaptability also was said to have served as an encouragement, with participants often drawing from their past as motivation in order to push through present difficulties.

Throughout the interviews, participants frequently offered advice to fellow military children both voluntarily, and when elicited by the researchers with specific questions. The advice centered on being flexible, being willing to be adaptable, and changing with the situations. In general, participants wanted to encourage fellow MKs not to lose hope as they endure the hardships because, in the end, they can and will make it through. Peter best summarized the idea of reward following endurance: "Stick with it. Stick through it... the things 
that you learn through the hardships are definitely worth it in the end." Holistically, participants related feeling that, although life presented them with many different challenges, they had become stronger people because of these challenges. When asked whether or not they had significant regrets about life as a MK, participants smiled and universally told us, "None."

\section{Overall Positive Experience}

Although the research participants shared a variety of different trials, struggles, and challenging life issues, they most often described their upbringing positively. The MKs described their childhood with adjectives such as "fun," "adventurous," "awesome," and "great." As an illustration, when asked what word came to mind when thinking of his upbringing, Jeff hesitated, and then stated: "I would honestly use awesome. Just because there's a lot of cool experiences from it." Participants commonly reported that the military provided positively unique experiences. They expressed an appreciation for their upbringing and the opportunities that it provided them, especially in regards to travel. Participants frequently told researchers about how many states in which they had been, countries they had seen, and other attractions they had enjoyed. One clear example of this phenomenon was when Gloria excitedly stated:

It's just so crazy to be like, oh you know 45 states and all of these national parks and so many family trips, and none of those things would have even been possible without the military, the military life; so it's been totally worth it.

Overall, the MKs spoke with positive tones (including non-verbals) and participants generally expressed sanguine attitudes regarding their upbringing. In retelling their stories, despite the hardships, participants frequently shared joyful stories and laughs. We concluded that MKs feel as though life may have been hard at times, but it was the life they were glad to have lived. 


\section{Discussion}

The overall purpose of this study was to gain a better understanding of the ways MKs perceive that their unique upbringing has influenced their development and current state. Participants described feeling as though their lives involved hardships at times but they also perceived themselves as becoming stronger because of the trials. When asked to describe themselves, participants chose adjectives that described their flexibility and constant adapting in a positive light. Participants also described possessing opportunities for unique experiences due to their military upbringing. Consistent with Charrand and colleagues (2008), findings from our study showed military deployments to have potentially indelible impacts on MKs. As an added dynamic, however, we found that the timing of parental deployment had a noted effect on how the child viewed his/her upbringing. Participants whose parents deployed during their teen years were much more likely to describe the difficulties associated with the deployment. Integrated with the results of Mustillo and colleagues (2016), we conclude that parental deployment possesses multiple variables, such that how MKs respond likely is more idiosyncratic — rather than fitting a definable sociological pattern.

Some changes during parental deployment were noted to be positive, such as interdependence on siblings, while others were described negatively, such as overreliance on the non-deployed parent. Hider's (1958) classic attribution theory regarding internal and external attribution may be applied to the described dynamics: MKs become challenged with findingtheir-own way psychologically as respective roles change. MKs with more fluid personal constructs may find fewer struggles in adapting than do MKs who possess more rigid constructs. 
Additionally, Kelly's (1967) classic covariation model of attribution theory involving consensus, distinctiveness, and consistency also may help to interpret the MK's noted self-perceptions. In particular, MKs may rely on multiple-necessary-causes (e.g., self-reliance was effective so it should continue) or multiple-sufficient-cause (e.g., family equilibrium is desired but has been disrupted when the parent returns stateside).

Bowlby's (1958) attachment theory may also be related to the findings of our study. Making meaningful connections with parents and bonding is a significant developmental task. Attachment at various stages in pre-elementary and elementary school years has shown to have predictive influence in later behaviors, including antisocial tendencies (Ainsworth, 1973). Participants from our present study indicated feeling extra connections with their mothers when fathers were deployed overseas. While we do not have sufficient information from the transcript interviews to draw particular implications for all $\mathrm{MKs}$, we make special note here and believe the construct is worth future exploration. Examples of how MKs could be influenced by parental bonding issues include self-regulation (Burnette, O’Boyle, VanEpps, Pollack, \& Finkel, 2013), moral reasoning, emotional regulation, and some types of executive functioning (Hinnant, Nelson, O’Brien, Keane, and Calkins, 2013).

Another theory that may explain why the MKs experienced positive personal growth despite the increased demands of their unique upbringing is the Resiliency Theory (Fergus \& Zimmerman, 2005). This theory focuses on the positive aspects of a child's life that may function as protective factors (Black et al. 2016), and as such, mitigate the adversity in the child's life in order to prevent further development along a risk trajectory, and help the child develop resilience (Zimmerman, 2013). The two main protective factors that lead to resilience development are resources, which are external factors such as parental and community support, 
and assets, which are personal factors such as self-esteem and self-efficacy (Fergus \& Zimmerman, 2005). Many of the MKs expressed a strong sense of parental support (resources) and self-esteem (asset) which may have aided in their development of adaptability in spite of negative circumstances, which is closely tied to the psychological concept of resiliency. Rutter's (1987) Challenge Model of Resiliency may also help explain why the MKs felt that their challenging upbringing led to positive long-term outcomes. In this model, Rutter suggests that if teens are exposed to modest "risks" early on they may be less vulnerable to future challenges, as this initial inoculation forces the development of coping strategies that can be employed to help during future adverse circumstances. Overall, these theories suggest that our MKs developed adaptability not in spite of their difficult upbringings, but perhaps because of them.

\section{Implications}

We believe the findings of our present study are applicable to a number of different groups, including military parents, military psychologists, social workers, school counselors, and teachers. Each group has connections with MKs, at various life stages, and findings from the present research are useful on multiple levels. Better understanding the dynamics experienced by MKs could help people provide better empathy, offer more effective services, make better decisions, and generally relate to MKs in enhanced ways. Understanding both the aversive and positive outcomes of their rearing may help reframe their circumstances in a more positive light. Additionally, this research suggests that military kids are aware of their situation and may be best able to help enhance existing programs designed to help them. Taking affirmative steps with some MKs in order to help them cope with various life struggles may be warranted in some cases. Offering support services, both formal and informal, may be helpful to many MKs during key developmental periods (particularly when parents deploy). 
The participants in the present study described the experience of self-expression (sharing personal aspects regarding their developmental years) as being very helpful when relating their stories with someone who was willing to listen. Apart from the present research study, it may be beneficial for MKs to discuss their life experiences in both formal and informal settings in a variety of potential contexts. Social support networks for MKs on various college campuses, for example, may be beneficial for some students making the transition from living in base-/military community-housing to living in dormitory or fraternity/sorority settings. Clubs or other cohesion groups may be beneficial as experienced MK students can share in meaningful ways how they transitioned from military to university milieus. Throughout the interviews, participants spontaneously shared advice they had for younger MKs, including being adaptable through the changes, maintaining close relationships with siblings, and making the most of the military experience. Organized and informal MK groups may be helpful supports to peer college students as well as high school students who are readying themselves for the upcoming transition.

\section{Limitations and Future Research}

Good research practice includes noting the limitations of all research studies (Shipman, 2013) and we close the present article noting these elements within our study. First, all qualitative research is context-specific (Hannes, 2016) and this principle applies to our present study. We have shown that our study possesses viable elements relating to credibility and trustworthiness and, as such, we believe the reported findings aptly reflect the perspectives of the research participants. The degree to which the findings possess transferability and dependability, however, is unknown and, in qualitative research protocol, such confirmation is discovered through replication (Firmin, 2008). That is, as qualitative studies (such as our present one) are replicated across cultures, time, samples, and contexts - patterns emerge and researchers are 
better able to assess patterns, unique cases, and commonalities. The findings of our present study are viewed, therefore, within this larger structure of MK research.

Another limitation of our study is that research participants were mostly Caucasians. Consequently, future qualitative research in this domain should include samples of individuals from minority cultures. Further, researchers should also consider comparing potential differences that might exist among MKs with various cultural and/or ethnic backgrounds. Given the rich diffusion that different cultural groups bring to the MK experience, contrasting any potential findings among such groups would be interesting.

Third, the sample of participants in our present study were students at a Midwestern, selective, comprehensive university. Consequently, future research should include samples from divergent academic contexts such as research universities, community colleges, specialized institutions, regional state colleges, and military academies. Given the rich diffusion that exists among students at such institutions of higher education, contrasting potential findings would be interesting.

Because MKs from the present sample were college students, future researchers should replicate our present study with MKs who are not in college at the time of data collection. Comparing any potential differences in perspectives between non-college students and the perspectives related in our study would be interesting. And finally, quantitative researchers should consider utilizing the findings from our present study when generating national surveys pertaining to MK experiences. As noted earlier, quantitative research tends to answer "how many" type of questions; future survey research potentially would help extend our study's generalizability and expand the breadth of understanding regarding MK experiences. 


\section{Conclusion}

It has previously been reported that military kids may have unique difficulties and risks due to their uncommon childhood, however few studies have assessed the impact of their childhood on their college years. The current study is one of the first to report on the perception of military kids in regards to their upbringing, such as frequent moves and parental deployments. We sought to explore the shared, lived experience of military kids through qualitative interviewing, which a particular focus on the perceived effects of their childhood on their current state with regards to affect, behavior, and cognition.

Overall findings suggest that although the deployments were challenging for the family, the experiences also led to positive outcomes such as closer family relationships with mothers and siblings, increased adaptability, and positive new experiences. The findings of this study also suggest that better understanding the remarkable experiences of military kids may help parents/teachers/providers serve them more effectively and better provide for their individual needs. Finally, the findings also suggest that the greatest support for military kids may be found in others with similar experiences, and this should be considered when designing programs to help this population, both within and outside of the military. 


\section{References}

Ainsworth, M. D. S. (1973). The development of infant-mother attachment. In B. Cardwell \& H. Ricciuti (Eds.), Review of child development research (Vol. 3, pp. 1-94) Chicago, IL: University of Chicago Press.

Alfano, C., Lau, S., Balderas, J., Bunnell, B., \& Beidel, D. (2016). The impact of military deployment on children: Placing developmental risk in context. Clinical Psychology Review, 42, 17-29.

Alvesson, M. (2011). Interpreting interviews. Thousand Oaks, CA: Sage.

Berg. B. L. (2012). Qualitative research for the social sciences $\left(8^{\text {th }}\right.$ ed.). Boston, MA: Allyn \& Bacon.

Black, M. M., Fernandez-Rao, S., Hurley, K. M., Tilton, N., Balakrishna, N., Harding, K. B., .. . Nair, K. M. (2016). Growth and development among infants and preschoolers in rural India: Economic inequities and caregiver protective/promotive factors. International Journal of Behavioral Development, 40(6), 526-535.

Bowlby, J. (1958). The nature of the child's tie to his mother. International Journal of Psychoanalysis, 39, 350-371.

Burnette, J., O'Boyle, E., VanEpps, E., Pollack, J., \& Finkel, E. (2013). Mind-sets matter: A meta-analytic review of implicit theories and self-regulation. Psychological Bulletin, $139,655-701$

Chartrand, M. M., Frank, D. A., White, L. F., \& Shope, T. R. (2008). Effect of parents' wartime deployment on the behavior of young children in military families. Archives of Pediatrics \& Adolescent Medicine, 162, 1009-1014. 
Chenail, R. J. (2012a). Conducting qualitative data analysis: Reading line-by-line, but analyzing by meaningful qualitative units. The Qualitative Report, 17, 266-269.

Chenail, R. J. (2012b). Conducting qualitative data analysis: Managing dynamic tensions within. The Qualitative Report, 17, 1-6.

Creswell, J. (2012). Education research: Planning, conducting, and evaluating quantitate and qualitative research. Boston, MA: Pearson.

Clever, M., \& Segal, D. R. (2013). The demographics of military children and families. Future of Children, 23, 13-39.

DeCuir-Gunby, J. T., Marshall, P. L., \& McCulloch, A. W. (2011). Developing and using a codebook for the analysis of interview data: An example from a professional development research project. Field Methods, 23(2), 136-155.

Defense Manpower Data Center (2006). 2006 Survey of Active-Duty Spouses: Administration, Datasets, and Codebook. Arlington, VA: Defense Manpower Data Center

Firmin, M. (2006). External validity in qualitative research. In M. Firmin \& P. Brewer (Eds.), Ethnographic \& qualitative research in education Vol. 2 (pp. 17-29). New Castle, UK: Cambridge Scholars Press.

Firmin, M. (2008). Replication. In L. Givens (Ed.), Sage encyclopedia qualitative research methods (Vol. 2; pp. 754-755). Thousand Oaks, CA: Sage.

Flake, E., Davis, B., Johnson, P., \& Middleton, L. (2009). The psychosocial effects of deployment on military children. Journal of Developmental \& Behavioral Pediatrics, 30, 271-278. 
Flick, V. (2006). An introduction to qualitative research (3rd ed.). Thousand Oaks, CA: Sage.

Fergus, S., \& Zimmerman, M. A. (2005). Adolescent resilience: A framework for understanding healthy development in the face of risk. Annual Review of Public Health, 26(1), 399-419.

Gay, L.R., Mills, G.E., \& Airasian, P. (2009). Educational research (9th ed.). Upper Saddle River: NJ: Pearson.

Gorman, G. H., Eide, M., \& Hisle-Gorman, E. (2010). Wartime military deployment and increased pediatric mental and behavioral health complaints. Pediatrics, 126(6), 10581066

Guest, G., Bunce, A., \& Johnson, L. (2006). How many interviews are enough? An experiment with data saturation and variability. Field Methods, 18, 59-82.

Hannes, K. (2016, May). Strengthening the qualitative research agenda in Europe. Paper presented at the International Conference of Qualitative Research, Champaign, IL.

Harsh, S. (2011). Purposeful sampling in qualitative synthesis. Qualitative Research Journal, 11, $63-75$.

Hinnanta, J. B., Nelsonb, J., O'Brienc, M., Keaned, S., \& Calkinsc, S. (2013). The interactive roles of parenting, emotion regulation and executive functioning in moral reasoning during middle childhood. Cognition and Emotion, 27, 1460-1468.

Kelley, H. H. (1967). Attribution theory in social psychology. In D. Levine (Ed.), Nebraska Symposium on motivation (Volume 15, pp. 192-238). Lincoln, NE: University of Nebraska Press.

Lester, P., Peterson, K., Reeves, J., Knauss, L., Glover, D., Mogil, C., . . Beardslee, W. (2010). The long war and parental combat deployment: Effects on military children and at home 
spouses. Journal of the American Academy of Child \& Adolescent Psychiatry, 49, 310320.

Maxwell, J. A. (2012). Qualitative research design (3 ${ }^{\text {rd }}$ ed.). Thousand Oaks, CA: Sage.

Mero-Jaffe, I. (2011). 'Is that what I said?' Interview transcript approval by participants: An aspect of ethics in qualitative research. International Journal of Qualitative Methods, 10, 231-247.

Moustakas, C. E. (1994) Phenomenological research methods. Thousand Oaks, Calif: Sage.

Mustillo, S., Wadsworth, S., \& Lester, P. (2016). Parental Deployment and well-being in children: Results from a new study of military families. Journal of Emotional and Behavioral Disorders, 24, 82-91.

Neuman, W. L. (2006). Social research methods: Qualitative and quantitative approaches. ( $6^{\text {th }}$ ed.). Boston, MA: Allyn \& Bacon.

Newman, I., Ridenour, C. S., Newman, C., Smith, S., \& Brown, R.C. (2013). Detecting low incidents effects: the value of mixed methods. Research designs in low-n studies. Midwestern Educational Researcher, 25, 31-46.

Office of the Deputy Assistant Secretary of Defense. (2013). 2013 Demographics: Profile of the military community. Retrieved from http://download.militaryonesource.mil/12038/MOS/Reports/2013-DemographicsReport.pdf.

Oppong, S. H., (2013). The problem of sampling in qualitative research. Asian Journal of Management Sciences and Education, 2, 202-210. 
Rapport, F., Storey, M., Porter, A., Snooks, H., Jones, K., Peconi, J., ... \& Russell, I. (2013). Qualitative research within trials: Developing a standard operating procedure for a clinical trials unit. Trials, 14, 1-8.

Rodgers, B. (2008). Audit trail. In L. Given (Ed.), The Sage encyclopedia of qualitative research methods (Vol 1; pp. 43-44). Thousand Oaks, CA: Sage.

Sandoz, E. K., Moyer, D. N., \& Armelie, A. P. (2015). Psychological flexibility as a framework for understanding and improving family reintegration following military deployment. Journal of Marital and Family Therapy, 41, 495-507.

Shipman, M. (2014). The limitations of social research ( $4^{\text {th }}$ ed.). New York, NY: Routledge.

Silverman, D. (2012). Interpreting qualitative data (4 ${ }^{\text {th }}$ ed.). Thousand Oaks, CA: Sage.

Sin, S. (2010). Considerations of quality in phenomenographic research. International Journal of Qualitative Methods, 9, 305-319.

Zimmerman, M. A. (2013). Resiliency theory: A strengths-based approach to research and practice for adolescent health. Health Education \& Behavior, 40(4), 381-383. 